\title{
OTR INTERFEROMETRY DIAGNOSTIC FOR THE A0 PHOTOINJECTOR*
}

\author{
G. Kazakevich", BINP, Novosibirsk, 630090, Russia \\ H. Edwards, R. Fliller, V. Lebedev, S. Nagaitsev, R. Thurman-Keup, \\ FNAL, Batavia, P.O. Box 500, IL, U.S.A.
}

\section{Abstract}

OTR interferometry (OTRI) is an attractive diagnostic for investigation of relativistic electron beam parameters. The diagnostic is currently under development at the A0 Photoinjector. This diagnostic is also applicable for NML accelerator test facility that will be built at Fermilab. The experimental setup of the OTR Interferometer for the FNAL A0 Photoinjector is described in the report. Results of simulations and measurements are presented and discussed.

\section{INTRODUCTION}

A charged particle passing through the boundary of two medias having different permittivities generates Transition Radiation (TR), [1]. The TR is caused by a variation of the particle electric field with the variation of the permittivity. The TR for relativistic particles has a wide spectrum with a significant portion in the optical range. The Optical Transition Radiation (OTR) is widely used for a beam profile and a beam size monitoring. Moreover, OTR can be used to characterize the energy, energy spread and transverse angles in the beam by using the interference of the OTR from two thin films [2] inserted in the beam trajectory. This method has been applied in a number of works [3-5] demonstrating good results and good agreement between measurements and calculations. In this paper we present a simulation of the interference patterns for an experimental setup developed for A0 Photoinjector.

\section{OTRI PATTERN SIMULATION}

The setup for the OTR interferometry (OTRI) at the A0 Photoinjector employs two thin films, displaced by a distance $D$ and mounted in the accelerator beamline.

The electron beam incident angle on the film plane is $45^{0}$. The first film (A) is optically transparent; second one has a mirror-reflecting metal surface, Fig. 1, [6].

For optical materials traditionally used in the OTR interferometry the total internal reflection angle $\theta_{r}=\sin ^{-1}\left(n^{-1}\right) \approx 0.65-0.7 \mathrm{rad}$, so the main portion of the forward OTR generated on the entrance in the $\mathbf{A}$ film having $\pi / 4=0.78 \mathrm{rad}$. inclination to the beam axis disappears. Only the photons radiated with relatively big negative angles $<-0.1 \mathrm{rad}$ contribute to the total forward OTR; their contribution drops very rapidly with an increase of the energy of the electrons.

\footnotetext{
* Work supported by Fermi Research Alliance LLC. Under DE-AC0207 CH1 1359 with the U.S. DOE

"kazakevi@,fnal.gov
}

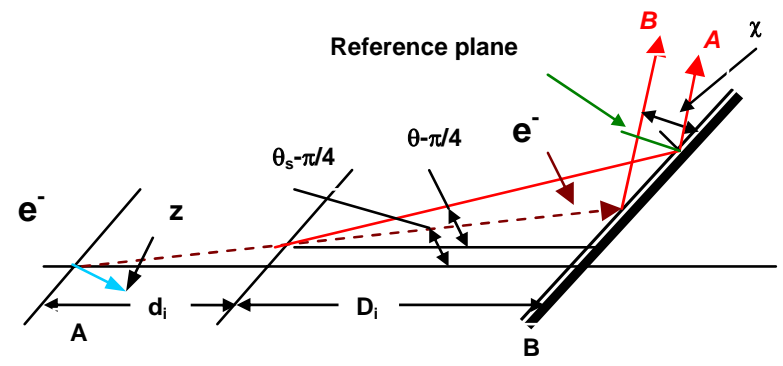

Fig. 1. Layout using transparent film (A) with thickness $d$ and mirror-reflecting film (B) at the oblique incidence of the particles. For considered OTR TM and TE waves the electric field vectors are in plane of this figure and perpendicular to the plane, respectively. The A film thickness is shown magnified. $\mathrm{Z}$ is a normal to the film.

For the energy of the A0 Photoinjector $(\sim 16 \mathrm{MeV})$ the contribution is approximately a few percent and one can omit it in the forward radiation generated by electrons passing through the A film.

Intensity in the forward OTR TM wave generated on the second boundary of the A dielectric film is described by expression first derived by V. Pafomov, [7]:

$I_{F d T M}(\theta, \varphi, \omega)=\frac{e^{2} \beta_{z}^{2} \cdot \cos ^{2} \theta_{z} \cdot|1-\varepsilon|^{2}}{\pi^{2} c\left[\left(1-\beta_{x} \cos \theta_{x}\right)^{2}-\beta_{z}^{2} \cos ^{2} \theta_{z}\right]^{2} \sin ^{2} \theta_{z}} \times$
$\left|\frac{\left(1-\beta_{z} \sqrt{\varepsilon-\sin ^{2} \theta_{z}}-\beta_{z}^{2}-\beta_{x} \cos \theta_{x}\right) \sin ^{2} \theta_{x}+\beta_{x} \beta_{z} \cos \theta_{x} \sqrt{\varepsilon-\sin ^{2} \theta_{x}}}{\left(1-\beta_{x} \cos \theta_{x}-\beta_{z} \sqrt{\varepsilon-\sin ^{2} \theta_{z}}\right) \cdot\left(\varepsilon \cos \theta_{z}+\sqrt{\left.\varepsilon-\sin ^{2} \theta_{z}\right)}\right.}\right|^{2}$

Here: $\varepsilon(\omega)$ is the permittivity of the dielectric film, $\theta$ is the angle between the normal to the film and wave vector of the OTR photon, $\beta_{z}=\beta \cos \psi, \beta_{x}=\beta \sin \psi, \psi$ is the angle of incidence of the electrons; the directional cosines of the wave vector are determined as: $\cos \theta_{x}=\sin \theta \cdot \cos \varphi ; \cos \theta_{z}=\cos \theta$. In the optical range $\varepsilon_{\omega} \approx$ const. So the intensity distribution does not depend on $\omega$.

Following Reference [7] one obtains intensity in the backward TM wave of the OTR, $I_{B m T M}(\theta, \varphi, \omega)$, on the boundary vacuum-metal of the $\mathbf{B}$ film. The expression differs from one written above by sign of $\beta_{z}$, the permittivity of the dielectric $\varepsilon$ must be used the imaginary part of permittivity for metal: $\varepsilon_{m}(\omega) \approx i \cdot 4 \pi \sigma / \omega$, where $\sigma$ is electrical conductivity of metal. In optical range $\sigma / \omega>>1$ and $I_{B m T M}(\theta, \varphi, \omega) \approx I_{B m T M}(\theta, \varphi)$

For the described setup main contributions in the interference pattern are given by the TM component of 
the forward OTR wave generated on the dielectricvacuum boundary of the A (transparent) dielectric film and TM component of the backward OTR wave generated on vacuum-metal boundary of the B film (mirrorreflecting metal), [6].

Obviously the TE waves only at $\varphi \cong \pi / 2$ will contribute to the interference with the considered TM waves. The contribution is noticeable at the angle $\theta \cong \pi / 4$ as $I_{F d T M}(\pi / 4, \varphi)=0$ and $I_{B m T M}(\pi / 4, \varphi)=0$.

Based on the above concepts we consider for sake of simplicity the superposition of the forward OTR TM wave generated by the electrons crossing the $\mathbf{A}$ film:

$F_{A}(\theta, \varphi, \lambda) \approx \sqrt{I_{F d T M}(\theta, \varphi)} \exp \left(i \cdot \Phi_{A}(\theta, \lambda)\right)$, and the backward OTR wave, generated by the electrons crossing the metal-coated film $\mathbf{B}$ :

$$
B_{B}(\theta, \varphi, \lambda) \approx \sqrt{I_{B m T M}(\theta, \varphi)} \exp \left(i \cdot \Phi_{B}\left(\theta, \theta_{s}, \lambda\right)\right) .
$$

Here: the phase factor $\Phi_{A}(\theta, \lambda)$ is expressed as:

$$
\Phi_{A}(\theta, \lambda)=\left[\frac{2 \pi}{\lambda} \cdot\left[\frac{D_{i}}{\cos \alpha}\left(1+\frac{\tan \alpha}{1-\tan \alpha}\right)\right]\right],
$$

the phase factor $\Phi_{B}\left(\theta, \theta_{s}, \lambda\right)$ is expressed as:

$\Phi_{B}\left(\theta, \theta_{s}, \lambda\right)=\left[\frac{2 \pi \cdot D_{i}}{\lambda} \cdot\left[\frac{1+\frac{\tan \alpha_{s}}{1-\tan \alpha_{s}}}{\beta \cos \alpha_{s}}+\sqrt{2}\left(\frac{\tan \alpha}{1-\tan \alpha}-\frac{\tan \alpha_{s}}{1-\tan \alpha_{s}}\right) \cdot \cos \chi\right]\right.$

$\lambda$ is a wavelength on which the interference is measured, $\alpha=\theta-\pi / 4, \alpha_{s}=\theta_{s}-\pi / 4, \chi(\theta)=\pi / 2-\theta, D_{i}=\sqrt{2} D$. The minimum value of the interferometer base $D$ is determined by the expression: $D \geq \lambda /\left(\theta^{2}+\gamma^{-2}\right)$.

In the two-film interferometer with the base $D>>d$, the multiple scattering of the electrons in the A film strongly affects the interferogram. The effect was calculated assuming Gaussian distribution of the scattered angles for the multiple scattering of the electrons passing through the interferometer. For a distribution $l$ of scattering angles $\theta_{s l}$ the partial intensities in the interfering waves were calculated. The common intensity was calculated as a sum of partial intensities at various scattering angles $\theta_{s l}$ considering the corresponding probabilities $\eta_{l}$ of the scattering angle.

The one dimensional projection of the r.m.s angle of multiple scattering in the film having the thickness $d$ was calculated using following expression, [8]:

$\theta_{s}=\sqrt{\left\langle\theta_{0}^{2}\right\rangle} \cong \frac{13.6 \mathrm{MeV}}{\beta \cdot c p} z \cdot \sqrt{\frac{x}{X_{0}}} \cdot\left[1+0.038 \ln \left(\frac{x}{X_{0}}\right)\right]$

where: $x=\rho \cdot d, \rho$ is the film density, $X_{0}$ is the radiation length of the film material. The expression underestimates contribution of the scattering by big angles in comparison with method based on the Moliere theory and applicable for multiple scattering of the relativistic electrons. However, OTR photons for the discussed energy are radiated mainly with small angles. Because of that the described OTRI setup does not allow contribution of the OTR photons radiated by the electrons scattered by big angles. This allows using the expression for $\theta_{s}$ to calculate the r.m.s. angles of multiple scattering.

Finally, considering the bandwidth of a filter used to select the required wavelength, the angular distribution of the intensity of the interfering waves was calculated as:

$$
I_{E}(\theta) \approx \sum_{\lambda} \sum_{l}\left(\eta_{l} \cdot\left|F_{A}(\theta, \lambda)+B_{B S}(\theta, l, \lambda)\right|^{2}\right)
$$

Here: the subscript $S$ means consideration of the multiple scattering. The subscript $E$ in the resulting value of the intensity distribution points to the energy of electrons; summing over $\lambda$ means summing over the filter bandwidth.

\section{OTRI SETUP FOR AO PHOTOINJECTOR}

As the electron beam energy is tens of $\mathrm{MeV}$ in the existing OTRI setups optically transparent films as Mylar or Kapton are generally used. The technology of film manufacturing allows one to get the thickness of the films in the micrometer range. This is acceptable to provide relatively small r.m.s. angles of multiple scattering $(\sim 1$ mrad.) at the considered energy of the electron beam. However, effects of the permanent use of organic materials and in the facilities utilizing a high-current photo-gun and/or superconducting cavities currently is not studied in detail.

The application of mica sheets in the OTRI has been studied because of their high vacuum properties, though optical and mechanical properties of Mylar or Kapton are more suitable for this purpose. Note that the mica is birefringent crystal; both optical axes are in plane of cleavage, but the refractive indexes are quite close, [9], that is acceptable to obtain first orders of the fringes.

The calculated distributions of the OTRI intensity vs. the electron beam energy for mica films at $d=10 \mu \mathrm{m}$, various optical filters and value of the interferometer base are presented in Fig. 2 and Fig. 3.

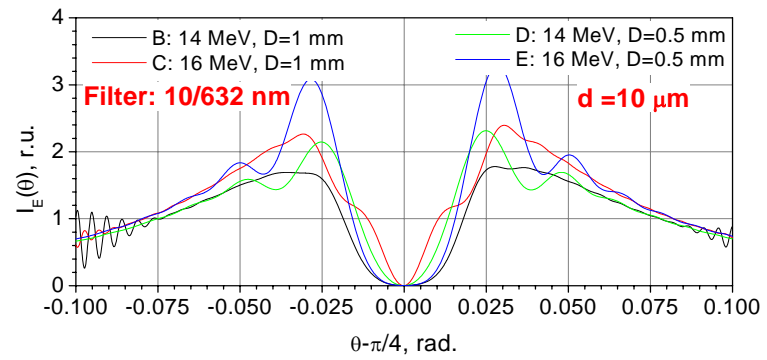

Fig. 2. Calculated profile of the OTRI angular distribution vs. the beam energy and the $D$ value for the $632 \mathrm{~nm}$ filter having the transmittance bandwidth of 10 nm.

The calculations allow us to choose an optimum of $D$, filter parameters for the given beam energy, and the film thickness to get best visibility, $v$, of the fringes. The visibility is determined as: $v=\left(I_{\max }-I_{\min }\right) /\left(I_{\max }+I_{\min }\right)$, where: $I_{\max }, I_{\min }$ are intensities in maximum and minimum 
of the fringes, respectively, and strongly depends on the r.m.s. angle of the multiple scattering.

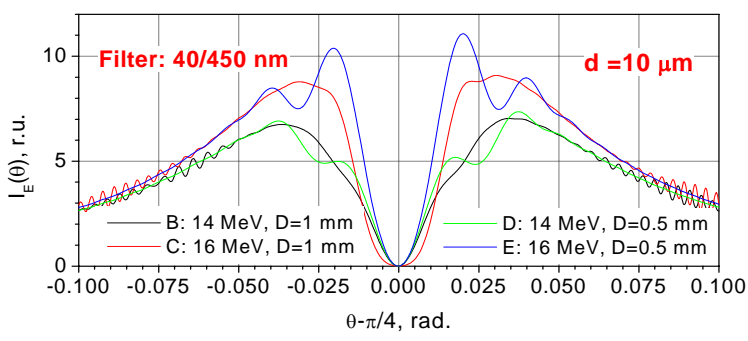

Fig. 3. Calculated profile of the OTRI angular distribution vs. the beam energy and the $D$ value for the $450 \mathrm{~nm}$ filter having the transmittance bandwidth of 40 $\mathrm{nm}$.

The visibility is significantly increased at lower r.m.s. angles of multiple scattering in the first film that implies smaller thickness of the first film.

The 10- $\mu \mathrm{m}$ thick, 1" diameter, colorless, optically transparent mica sheet has been used in the OTR interferometer as a film $\mathbf{A}$; film $\mathbf{B}$ was the same, but coated by aluminum, $\sim 80 \mathrm{~nm}$ thick. The spacer between the films provides parallelism of the films and pumping of the gap between them.

The $45^{\circ}$ incidence of the electrons on the A film plane was adjusted. The upstream triplet of quads provided focusing of the electron beam on the OTR interferometer films.

A movable collimating lens driven by a remote controlled picomotor was used to focus the interfering OTR on the photocathode of the MCP-based magnifier of the intensified CID (ICID) camera. A simple optical setup used in first experiments is shown in Fig. 4, sizes are in $\mathrm{mm}$.

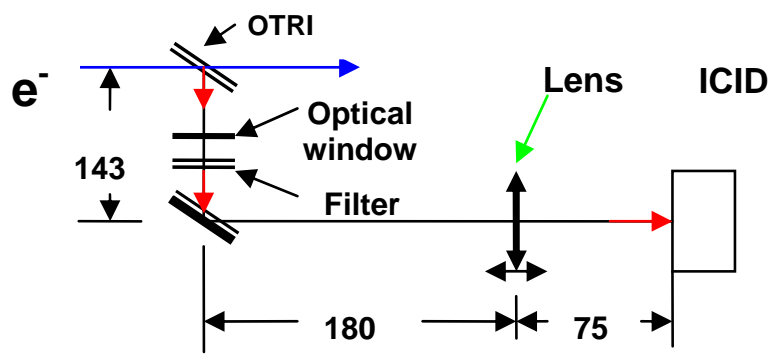

Fig. 4. Optical setup for OTRI experiments.

A gated ICID camera was used as an OTRI signal receiver providing magnification of the TR light in the narrow bandwidth of the used filters. The $160 \mathrm{~V}$ gating pulse having FWHM of $\sim 40 \mathrm{~ns}$ on the photo-cathode of the MCP magnifier provides strong suppression of the background caused by the dark current of the A0 photogun.

\section{EXPERIMENTAL RESULTS}

First experimental results were obtained at the beam energy of $14 \mathrm{MeV}$ and $16 \mathrm{MeV}$, bunch charge of $\sim 2 \mathrm{nC}$, 25 bunches per train, and the train repetition rate of $1 \mathrm{~Hz}$.
The OTRI patterns were measured with the filter having the central wavelength of $632 \mathrm{~nm}$ at the transmittance bandwidth of $\pm 5 \mathrm{~nm}$. Because of the narrow filter bandwidth and low sensitivity of the existing ICID to the filtered wavelength range the pattern images were averaged over 10 trains.

We have observed the expected difference in the interferometry pattern changing the energy of the electron beam from 14 to $16 \mathrm{MeV}$ using the above mentioned filter at $\mathrm{D}=1 \mathrm{~mm}$. The restored pattern profiles are shown in Fig. 5. In the figure are also shown the calculated pattern distributions which satisfactory fit the measured data.

Now improvements of the setup are in progress.

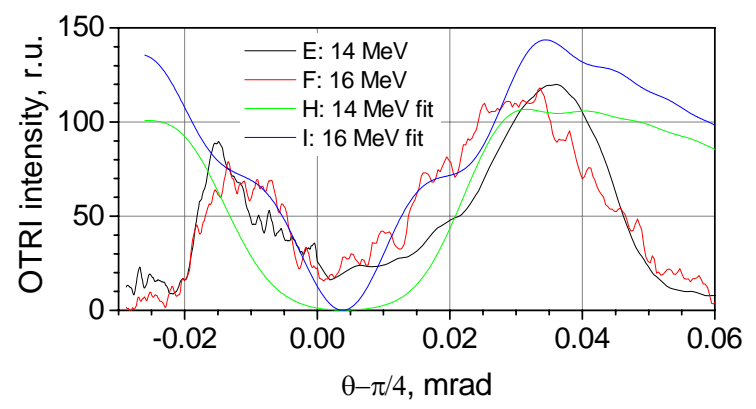

Fig. 5. Measured OTRI pattern profiles vs. the beam energy.

\section{SUMMARY}

Application of the mica sheets in the OTRI setup for A0 Photoinjector has been studied. Obtained results are adequate to considered simulation. Improvements of the setup now are in progress.

\section{REFERENCES}

[1] V.L Ginzburg and I.M. Frank, J. Exp. and Theoret. Phys., Vol. 16, pp. 15-21, 1946.

[2] L. Wartski et al., J. of Appl. Phys. Vol. 46, No. 8, 3644-3653, 1975.

[3] R.B. Fiorito and D.W. Rule, AIP Conference Proceed. Beam Instrumentation Workshop, V. 319, pp. 21-37, 1994.

[4] R.B. Fiorito and A.G. Shkvarunets, Proceedings DIPAC 2003, pp. 89-91, 2003.

[5] D.W. Rule et. al., NIM A296, (1990), 739-743,

[6] G. Kazakevich at al., Optical Transition Radiation Interferometry for A0 Photoinjector, FERMILABPUB-07-055-AD

[7] M.L. Ter-Mikaelian, High-Energy Electromagnetic Processes in Condensed Media, Wiley-Interscience, New-York, 1972.

[8] Review of Particle Physics, Phys. Rev. D, V 54, No 1, 132-139, 1996.

[9] E. Hecht, A. Zajak, "OPTICS", Addison-Wesley Publishing Company (1977 